\title{
Extra-virgin olive oil phenols block cell cycle progression and modulate chemotherapeutic toxicity in bladder cancer cells
}

\author{
ANDREA COCCIA $^{1,2}$, LUCIANA MOSCA ${ }^{3}$, ROSA PUCA ${ }^{1}$, GIORGIO MANGINO ${ }^{1}$, \\ ALESSANDRO ROSSI $^{2}$ and EUGENIO LENDARO ${ }^{1}$
}

\author{
${ }^{1}$ Department of Medical-Surgical Sciences and Biotechnologies, Sapienza University of Rome, \\ 04100 Latina; ${ }^{2}$ L.I.L.T. Italian League for The Fight Against Cancer - Latina Section, 04100 Latina; \\ ${ }^{3}$ Department of Biochemical Sciences, Sapienza University of Rome, 00185 Rome, Italy
}

Received April 29 2016; Accepted June 21, 2016

DOI: $10.3892 /$ or.2016.5150

\begin{abstract}
Epidemiological data indicate that the daily consumption of extra-virgin olive oil (EVOO), a common dietary habit of the Mediterranean area, lowers the incidence of certain types of cancer, in particular bladder neoplasm. The aim of the present study was to evaluate the antiproliferative activity of polyphenols extracted from EVOO on bladder cancer (BCa), and to clarify the biological mechanisms that trigger cell death. Furthermore, we also evaluated the ability of low doses of extra-virgin olive oil extract (EVOOE) to modulate the in vitro activity of paclitaxel or mitomycin, two antineoplastic drugs used in the management of different types of cancer. Our results showed that EVOOE significantly inhibited the proliferation and clonogenic ability of T24 and 5637 BCa cells in a dose-dependent manner. Furthermore, cell cycle analysis after EVOOE treatment showed a marked growth arrest prior to mitosis in the $\mathrm{G} 2 / \mathrm{M}$ phase for both cell lines, with the subsequent induction of apoptosis only in the T24 cells. Notably, simultaneous treatment of mitomycin C and EVOOE reduced the drug cytotoxicity due to inhibition of ROS production. Conversely, the co-treatment of T24 cells with paclitaxel and the polyphenol extract strongly increased the apoptotic cell death at each tested concentration compared to paclitaxel alone. Our results support the epidemiological
\end{abstract}

Correspondence to: Dr Andrea Coccia, Department of Medical-Surgical Sciences and Biotechnologies, Sapienza University of Rome, Corso della Repubblica 79, 04100 Latina, Italy

E-mail: andrea.coccia@uniroma1.it

Abbreviations: $\mathrm{BCa}$, bladder cancer; EVOOE, extra-virgin olive oil extract; MUFA, monounsaturated fatty acids; EPIC, European Prospective Investigation into Cancer and Nutrition; HTy, hydroxytyrosol; TY, tyrosol; DCF, 2',7'-dichlorofluorescein; TBS, Tris-buffered saline; BSA, bovine serum albumin

Key words: olive oil polyphenols, bladder cancer, paclitaxel, mitomycin, apoptosis, T24 cells, 5637 cells evidence indicating that olive oil consumption exerts health benefits and may represent a starting point for the development of new anticancer strategies.

\section{Introduction}

Bladder cancer $(\mathrm{BCa})$ is one of the most common cancers in Western countries and the first leading cause of death among urinary malignancies (1). The incidence and mortality rates of BCa vary substantially across countries, in part due to different risk factors. In 2007, a comprehensive review by the World Cancer Research Fund and the American Institute for Cancer Research concluded that established risk factors for $\mathrm{BCa}$ include tobacco consumption, Schistosoma haematobium infection, and both occupational and environmental exposure to carcinogens such as aromatic amines and polycyclic aromatic hydrocarbons and arsenic in drinking water (2).

The role of nutrition as a protective factor in the development of $\mathrm{BCa}$ remains unclear but many data indicate that a regular consumption of fruit and vegetables appears to be linked to a lower incidence of urothelial neoplasia (3). A recent study in a multiethnic cohort showed that the intake of vegetables and some related micronutrients such as vitamins A, C, E and carotenoids was inversely associated with $\mathrm{BCa}$ risk only in women (4). Ros et al showed that a higher plasma carotenoid concentration was associated with a lower incidence of $\mathrm{BCa}$, suggesting that specific compounds in fruit and vegetables may exert protective effects on BCa risk (5). Moreover, data of the European Prospective Investigation into Cancer and Nutrition (EPIC) study, found an inverse association between the dietary intake of flavanols and lignans and the risk of $\mathrm{BCa}(6)$.

Epidemiological evidence and many case-control studies strongly support the hypothesis that adherence to the Mediterranean diet reduces cancer risk and in particular olive oil consumption is inversely related to cancer prevalence $(7,8)$. Olive oil is the main dietary fat of the Mediterranean area and its health-promoting properties are well assessed by numerous studies (9-12). Historically, the beneficial effects of olive oil intake have been attributed to the high concentration of monounsaturated fatty acids (MUFAs) such as oleic acid that represents the main component. However, other oils rich in 
MUFA, derived from the seeds of soybean or rapeseed, do not exert the same health benefits as extra-virgin olive oil (EVOO). In the last few years, attention has been focused on the minor phenolic fraction mainly constituted of a complex mixture comprising at least 36 distinct compounds (10). The most represented phenolic molecules in EVOO are secoiridoids, such as oleuropein and ligstroside, and phenolic alcohols, such as hydroxytyrosol (HTy) and tyrosol (TY), accounting for $\sim 90 \%$ of total phenols. The remaining $10 \%$ of the mixture is mainly constituted by flavonoids and lignans. Polyphenols have well-known antioxidant, anti-inflammatory, cardioprotective, anti-atherogenic, antithrombotic, neuroprotective and anticancer activities (13-15). Recent findings suggest that in low amounts, polyphenols may exert pharmacological activity within cells. In particular, polyphenols have the potential to modulate intracellular signaling cascades, to affect gene expression, to interact with mitochondria and to induce antioxidant enzymes as well as to inhibit the expression of enzymes involved in the generation of free radicals (16). By affecting such pathways they have the ability to control cell survival, death and differentiation, and to exhibit marked anti-inflammatory activity via modulation of the expression of pro-inflammatory genes mainly acting through nuclear factor $-\kappa \mathrm{B}$ and mitogen-activated protein kinase signaling $(17,18)$. Owing to all of these properties, polyphenols exert anticancer effects through the modulation of genes and molecular signaling pathways associated with cell survival, cell cycle progression, cell growth arrest and apoptosis, as demonstrated in several tumor cell lines (19).

In a previous study, we demonstrated that very low doses of EVOO phenols inhibited the invasive ability of a BCa cell line by modulating the expression of MMP2 (20). The aim of the present study was to investigate the antiproliferative activity of extra-virgin olive oil extract (EVOOE) on $\mathrm{BCa}$, with the attempt to clarify the biological mechanisms that trigger cell death. Moreover, we also evaluated the ability of low doses of EVOOE to modulate the in vitro activity of paclitaxel or mitomycin $\mathrm{C}$, two antineoplastic drugs used in the management of different types of cancer.

\section{Materials and methods}

Materials. Acetonitrile $\left(\mathrm{CH}_{3} \mathrm{CN}\right), n$-hexane, dimethylsulphoxide (DMSO), acetic acid, methanol, 3-(4,5-dimethylthiazol-2-yl)-2,5-diphenyltetrazolium bromide (MTT), paclitaxel, mitomycin $\mathrm{C}$ and propidium iodide (PI) were purchased from Sigma-Aldrich (St. Louis, MO, USA). T24 and 5637 BCa cell lines were purchased from CLS Cell Lines Service GmbH (Eppelheim, Germany). All media and sera for cell culture were obtained from Invitrogen (Carlsbad, CA, USA) and were endotoxin-free. 2',7'-Dichlorodihydrofluorescein diacetate $\left(\mathrm{H}_{2} \mathrm{DCFDA}\right)$ was from Molecular Probes, Inc. (Eugene, OR, USA). For the immunofluorescence experiment, anti- $\alpha$-tubulin antibody, TRITC-labeled phalloidin and anti-mouse FITC-conjugated secondary antibody were purchased from Sigma-Aldrich (cat. nos. t5168, p1951 and f0257, respectively). Protease inhibitor cocktail was purchased from Sigma-Aldrich (cat. no. 11873580001) while Bradford reagent, Any $\mathrm{kD}^{\mathrm{TM}}$ polyacrylamide gel, anti-mouse and anti-rabbit secondary peroxidase-conjugated antibodies were obtained from Bio-Rad Laboratories, Inc. (Hercules, CA, USA) (cat. nos. 1706515 and 1706516, respectively). Primary antibodies for PARP-1 and GAPDH were obtained from Cell Signaling Technology, Inc. (Danvers, MA, USA) (cat. nos. 9542 and 2118, respectively), caspase-3 was from Santa Cruz Biotechnology, Inc. (Dallas, TX, USA) (cat. no. sc-7148), and caspase-9 was from Sigma-Aldrich (cat. no. c7729).

Extraction of the olive oil phenolic fraction. EVOOE was obtained as previously described (21) with minor modifications, from three different lots of an endemic monocultivar olive oil (Olea europaea L. var. Itrana) within 1 month after production. Fifty milliliters of each oil sample was extracted with $150 \mathrm{ml}$ of $\mathrm{CH}_{3} \mathrm{CN} / \mathrm{H}_{2} \mathrm{O}(70: 30 \mathrm{v} / \mathrm{v})$. A defatting with $n$-hexane was performed to completely remove the lipid fraction. Aliquots of the raw hydrophilic extract were dried by SpeedVac and stored at $-80^{\circ} \mathrm{C}$ until use. The extract was dissolved in DMSO at a stock concentration of $10 \mathrm{mg} / \mathrm{ml}$ immediately before performing the experiments.

HPLC analysis of the phenolic fraction. The HPLC analysis of the extract was carried out with a Waters apparatus, equipped with a $600 \mathrm{~F}$ pump and pump controller, a Rheodyne injection valve with a 50- $\mu 1$ loop, a Symmetry300 column (C18 reversed-phase column, 4.6×250 mm, 5- $\mu \mathrm{m}$ particle size, thermostated at $25^{\circ} \mathrm{C}$, with a $3.9 \times 20 \mathrm{~mm}$ guard column of the same material matrix), and a Waters 2996 Photodiode Array Detector. The elution was performed at a flow rate of $1 \mathrm{ml} / \mathrm{min}$, with solvent $\mathrm{A}$ being $2 \%$ acetic acid and solvent $\mathrm{B}$ being methanol. The gradient consisted of an initial $5 \% \mathrm{~B}$ for $5 \mathrm{~min}$, to $50 \% \mathrm{~B}$ in $35 \mathrm{~min}, 100 \% \mathrm{~B}$ in $10 \mathrm{~min}, 100 \% \mathrm{~B}$ for $10 \mathrm{~min}$. Samples were filtered through a $0.45-\mu \mathrm{m}$ cellulose syringe filter before injection. Elution was monitored at $280 \mathrm{~nm}$ and peak identification was obtained by comparing retention times and spectral characteristics with those of authentic standards.

Cell cultures. T24 and 5637 cells (human urinary bladder carcinoma) were cultured in DMEM without phenol red supplemented with $10 \%$ FBS, 2 mM glutamine, $100 \mathrm{U} / \mathrm{ml}$ penicillin and $100 \mu \mathrm{g} / \mathrm{ml}$ streptomycin.

Cell proliferation assays. Cell viability was assessed using the dye MTT. The assay is based on the ability of living cells to convert MTT into an insoluble purple-colored formazan, whose amount is proportional to the number of living cells. Cells seeded in 96-well plates at a density of 5,000 cells/well were exposed to different concentrations of EVOOE, paclitaxel or mitomycin C. After $24 \mathrm{~h}$, the cells were treated with $20 \mu \mathrm{l}$ of a $5 \mathrm{mg} / \mathrm{ml}$ solution of MTT in PBS and incubated at $37^{\circ} \mathrm{C}$ for $4 \mathrm{~h}$. After discarding the medium, the formazan was extracted with DMSO, and absorbance was read at $570 \mathrm{~nm}$ with a reference at $690 \mathrm{~nm}$ with a Tecan Sunrise ${ }^{\mathrm{TM}}$ microplate reader.

Colony-forming ability. The cells were plated at a density of 20 cells $/ \mathrm{cm}^{2}$ in tissue culture Petri dishes and exposed to various concentrations of EVOOE. Cells were grown for 14 days with a medium renewal every 2 days. After 2 weeks the cells were fixed with ethanol, and stained with Giemsa stain to detect the colonies. 
Cell cycle analysis. Flow cytometric analysis of the cells was performed using an Accuri C6 flow cytometer (BD Biosciences, Oxford, UK). To analyze cell cycle distribution, the cells were initially treated with various concentrations of EVOOE for $24 \mathrm{~h}$, and were then collected by trypsinization, fixed in $75 \%$ absolute ethanol, washed in PBS, and resuspended in $1 \mathrm{ml}$ of PBS containing $0.5 \mathrm{mg} / \mathrm{ml}$ of RNase A and $0.01 \mathrm{mg} / \mathrm{ml}$ of PI in the dark for $30 \mathrm{~min}$ at room temperature. The percentage of cells in the sub-G1, G0/G1, S, and G2/M phases of the cell cycle was analyzed using the ModFit LT 3.0 software (Verity Software House, Inc., Topsham, ME, USA).

Immunofluorescence staining of fibrous actin ( $F$-actin) and $\alpha$-tubulin. Cells were plated at a density of $2 \times 10^{4}$ cells $/ \mathrm{cm}^{2}$ on a glass coverslip. After $24 \mathrm{~h}$, the media were removed and the cells were treated with media containing different doses of EVOOE for $4 \mathrm{~h}$. After the incubation, the media were discarded and the glass coverslips were fixed for $20 \mathrm{~min}$ at $-20^{\circ} \mathrm{C}$ in methanol. After fixation, the cells were rinsed three times with cold PBS and then permeabilized in PBS containing $0.5 \%(\mathrm{v} / \mathrm{v})$ Triton $\mathrm{X}-100$ for $5 \mathrm{~min}$, blocked in bovine serum albumin (BSA) 5\% (w/v) for $1 \mathrm{~h}$ and then incubated overnight at $4{ }^{\circ} \mathrm{C}$ with a human monoclonal anti- $\alpha$-tubulin antibody produced in mouse diluted at 1:1,000 in PBS containing $0.2 \%$ (w/v) Triton X-100. After incubation, the coverslips were rinsed three times in PBS and incubated for $45 \mathrm{~min}$ with a solution of TRITC-labeled phalloidin which specifically binds to F-actin and anti-mouse FITC-conjugated antibody. The cell nuclei were counterstained with DAPI and the specimens were mounted on glass slides. The images were captured with a Nikon ViCo. 2 video confocal microscope (Nikon, Inc., Garden City, NY, USA) at a magnification of x600.

Measurement of intracellular ROS generation. Intracellular ROS levels were evaluated by treating cells with $2 \mu \mathrm{M}$ $\mathrm{H}_{2}$ DCFDA for $1 \mathrm{~h}$ at $37^{\circ} \mathrm{C}$ in the presence of different doses of EVOOE. $\mathrm{H}_{2}$ DCFDA, the acetylated form of 2',7'-dichlorofluorescein (DCF), is not fluorescent until intracellular deacetylation and oxidation by peroxides (22). Fluorescent intensities were quantified by flow cytometry.

Western blot analysis. T24 and 5637 cells were seeded in $10-\mathrm{cm}$ Petri plates at a concentration of $1 \times 10^{6}$ cells/plate. After $24 \mathrm{~h}$, the cells were treated with different concentrations of EVOOE for an additional $24 \mathrm{~h}$. The cells were scraped in culture medium, washed twice with PBS, and finally lysed into $200 \mu$ l RIPA buffer [50 mM Tris, pH 8.0, $150 \mathrm{mM} \mathrm{NaCl}$, $1 \%$ NP-40, $0.5 \%$ Na-deoxycholate, and $0.1 \%$ sodium dodecyl sulfate (SDS)], containing a protease inhibitor cocktail. After $20 \mathrm{~min}$ on ice, the lysates were centrifuged at $20,000 \mathrm{x} \mathrm{g}$ for $10 \mathrm{~min}$. Protein concentration was determined with the Bradford reagent. The lysates ( $20 \mu \mathrm{g}$ of the total protein) were resolved on Any $\mathrm{kD}^{\mathrm{TM}}$ polyacrylamide gel and transferred onto PVDF membranes. Membranes were blocked for $1 \mathrm{~h}$ with 5\% BSA, dissolved in Tris-buffered saline (TBS) solution (10 mM Tris- $\mathrm{HCl}, \mathrm{pH} 7.5,150 \mathrm{mM} \mathrm{NaCl}$ ). Membranes were then successively incubated overnight at $4^{\circ} \mathrm{C}$ with primary human antibodies in TBST (TBS with $0.1 \%$ Tween-20) with $5 \% \mathrm{BSA}$ at the following dilution: PARP-1 1:1,000, caspase-9, caspase-3 1:500 and GAPDH 1:4,000. After washing in TBST solution, the secondary antibody [anti-rabbit or anti-mouse IgG horseradish peroxidase (HRP)-conjugated antibody], diluted at 1:10,000 in TBST/5\% BSA, was added for $50 \mathrm{~min}$ at room temperature. Signals were visualized by ECL reagent according to the manufacturer's instructions.

Statistics. Each assay was replicated at least four times, and statistical significance was determined using GraphPad Prism 4 statistical software package (GraphPad Software, Inc., San Diego, CA, USA). Data are expressed as means \pm SD. Comparison of the groups was carried out by one-way ANOVA followed by Bonferroni's post hoc test. Statistical significance was defined as $\mathrm{P}<0.05$.

\section{Results}

EVOOE chromatographic analysis. EVOOE was analyzed by HPLC-DAD to identify the main phenolic compounds present in the extract. Analyses revealed a chromatographic profile strictly similar to the one previously obtained for the same olive oil extraction (20) which was characterized by a large amount of secoiridoids such as the dialdehydic form of elenolic acid linked to TY ( $p$-HPEA-EDA; oleocanthal) and the dialdehydic form of elenolic acid linked to HTy (3,4-DHPEA-EDA; oleuropein aglycon, dialdehydic form), and of lignans such as pinoresinol, whereas the simple phenolic fraction was scarcely represented, with very low amounts of TY, HTy, vanillic acid, vanillin, $p$-coumaric and ferulic acids. The low content of simple phenols such as TY and HTy indicates that the olive oil samples were obtained by correct production procedures giving rise to a high quality EVOO (20).

EVOOE inhibits cell growth and clonogenic survival. The cytotoxic effect of EVOOE on BCa cells was evaluated by MTT assay after $24 \mathrm{~h}$ of exposure to the phenolic extract. As shown in Fig. 1A, EVOOE treatment decreased T24 cell viability in a dose-dependent manner with growth inhibition ranging from 40 to $\sim 90 \%$, at 25 and $100 \mu \mathrm{g} / \mathrm{ml}$, respectively and an $\mathrm{IC}_{50}$ of $32 \pm 3 \mu \mathrm{g} / \mathrm{ml}$. The viability of 5637 cells was also inhibited by the treatment in a dose-dependent manner with a higher $\mathrm{IC}_{50}$ of $55 \pm 7 \mu \mathrm{g} / \mathrm{ml}$. Proliferation of neither of the two cell lines was affected at doses $<10 \mu \mathrm{g} / \mathrm{ml}$ up to $72 \mathrm{~h}$ (data not shown).

We also examined the effect of EVOOE at subtoxic doses on the clonogenic survival of T24 and 5637 cells. This assay determines the ability of a cell to proliferate indefinitely, retaining its reproductive ability to form a large colony or a clone. Importantly, both cell lines showed a significant decrease $(\mathrm{P}<0.001)$ in the ability to form colonies starting at a dose of $5 \mu \mathrm{g} / \mathrm{ml}$ with a marked inhibition of clonogenic activity at $10 \mu \mathrm{g} / \mathrm{ml}$ (Fig. 1B).

EVOOE blocks the cell cycle progression at G2/M stage. To better understand the mechanism underlying the cell growth impairment by EVOOE, cell cycle progression was investigated. T24 and 5637 cells were treated with increasing doses of EVOOE, and cell cycle distribution analysis was then performed after $24 \mathrm{~h}$ of exposure. As shown in Table I, the G2/M population of the EVOOE-treated T24 cells increased from $11.1 \%$ of the control to $\sim 27 \%$ at EVOOE doses of 

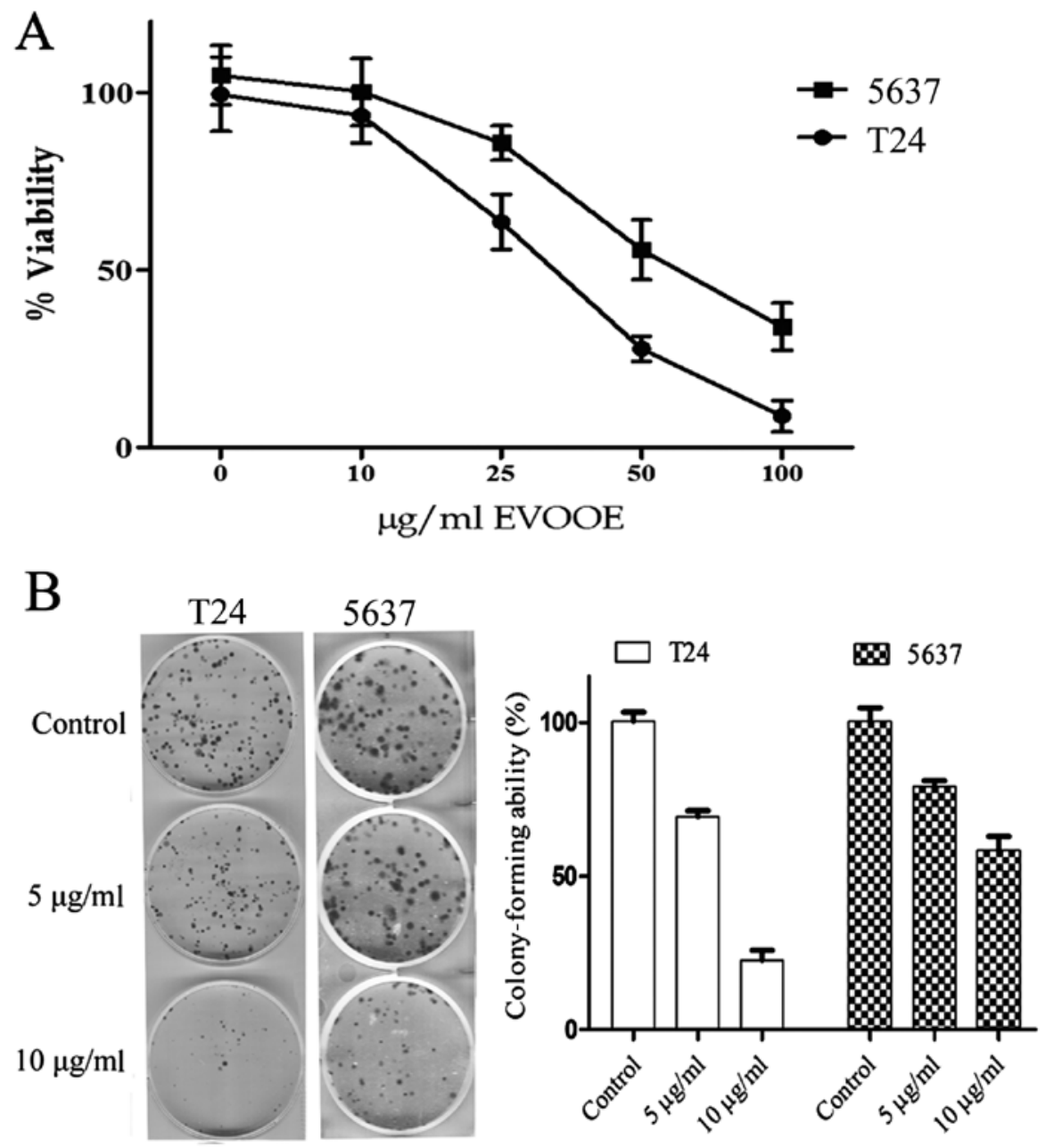

Figure 1. EVOOE inhibits cell growth. (A) Dose-response curve obtained with the MTT assay after $24 \mathrm{~h}$ of exposure to increasing doses of EVOOE. The data are presented as means $\pm \mathrm{SD}$. (B) Colony-forming ability assay. After 14 days of exposure to low doses of EVOOE $(\leq 10 \mu \mathrm{g} / \mathrm{ml})$, the colony-forming ability of both cell lines tested markedly decreased with a reduction in the number and dimension of the colony formed. The histogram represents the percentage of number of colonies formed after EVOOE treatment compared with the untreated control. The data are presented as means \pm SD. EVOOE, extra-virgin olive oil extract.

Table I. Percentage of cells in each phase of the cell cycle after $24 \mathrm{~h}$ of treatment with various doses of EVOOE. ${ }^{a}$

\begin{tabular}{|c|c|c|c|c|c|c|c|c|}
\hline \multirow{2}{*}{$\begin{array}{l}\text { EVOOE } \\
(\mu \mathrm{g} / \mathrm{ml})\end{array}$} & \multicolumn{4}{|c|}{$\mathrm{T} 24$ cells } & \multicolumn{4}{|c|}{5637 cells } \\
\hline & 0 & 10 & 20 & 40 & 0 & 10 & 20 & 40 \\
\hline Sub-G1 & $6 \pm 2$ & $8 \pm 0.5$ & $18 \pm 0.3^{b}$ & $28 \pm 3^{b}$ & $3 \pm 0.4$ & $2 \pm 0.5$ & $4 \pm 1$ & $5 \pm 1$ \\
\hline G1 & $72 \pm 4$ & $70 \pm 6$ & $41 \pm 4^{\mathrm{b}}$ & $30 \pm 2^{\mathrm{b}}$ & $60 \pm 5$ & $64 \pm 6$ & $59 \pm 5$ & $34 \pm 2^{b}$ \\
\hline$S$ & $12 \pm 3$ & $10 \pm 3$ & $16 \pm 4$ & $14 \pm 1$ & $24 \pm 1$ & $22 \pm 3$ & $16 \pm 0.7$ & $17 \pm 3$ \\
\hline $\mathrm{G} 2 / \mathrm{M}$ & $10 \pm 1$ & $12 \pm 4$ & $25 \pm 2^{b}$ & $28 \pm 4^{b}$ & $13 \pm 2$ & $12 \pm 0.7$ & $21 \pm 0.8^{\mathrm{b}}$ & $44 \pm 3^{b}$ \\
\hline
\end{tabular}

${ }^{\mathrm{a}}$ The data represent the mean percentage of cells \pm SEM and are calculated from three independent experiments. ${ }^{\mathrm{b}} \mathrm{P}<0.01$ compared to untreated control. EVOOE, extra-virgin olive oil extract.

20 and $40 \mu \mathrm{g} / \mathrm{ml}$. The treatment also induced a marked decrease in the G0/G1 population in a dose-dependent manner with a subsequent increase in sub-G1 particles (Fig. 2). This result revealed that EVOOE treatment caused marked accumulation of the G2/M population in the T24 cells that led to an accumulation of the sub-G1 fraction probably indicating induction of apoptosis. The cell cycle analysis of 5637 cells after EVOOE treatment revealed a similar behavior to that observed for the T24 cells, but with a consistently larger increase in the G2/M population at $40 \mu \mathrm{g} / \mathrm{ml}$ (28\% of T24 vs. $44 \%$ of 5637 cells). Notably, no increase in the sub-G1 fraction was observed for this cell line. 


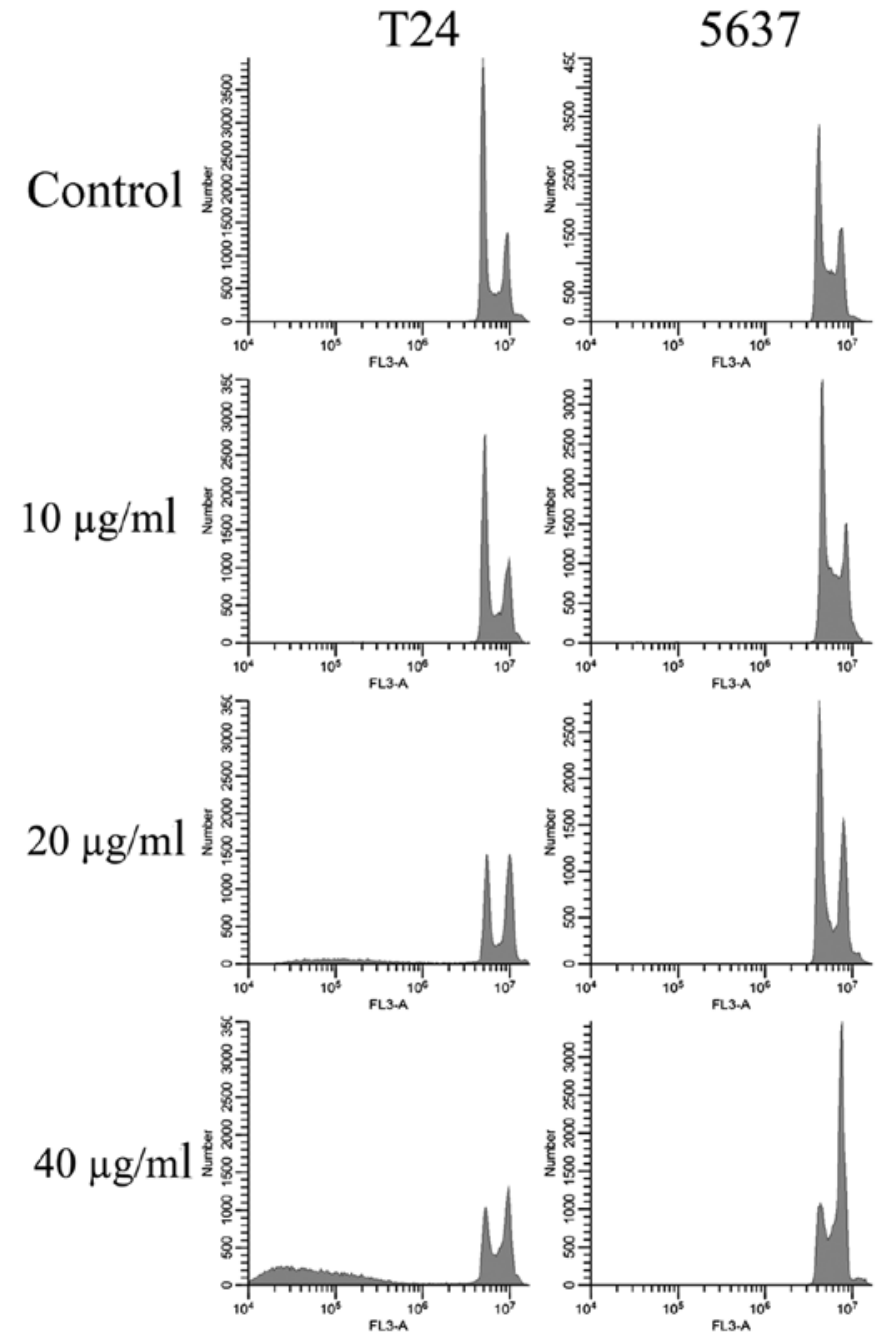

Short-time exposure to EVOOE alters cell morphology. Interestingly, a consistent change in cell morphology was noticeable in all the experiments performed. In order to explain this phenomenon, we tested the hypothesis that EVOOE could interfere with cytoskeleton remodeling and the subsequent mitotic process. After treatment with 20 and $40 \mu \mathrm{g} / \mathrm{ml}$ of EVOOE for $4 \mathrm{~h}$, the cells acquired a rounded morphology. In order to verify the alteration of the cytoskeleton, we performed immunofluorescence experiments using phalloidin (that bind to F-actin) and an antibody against $\alpha$-tubulin. As clearly visible from Fig. 3 , the treatment induced a change in T24 cell morphology with a marked variation of cytoskeleton network arrangement and a perinuclear accumulation of $\alpha$-tubulin.

EVOOE induces apoptosis only in T24 cells. Despite the cell cycle blockade in the $\mathrm{G} 2 / \mathrm{M}$ phase that occurred in both cell lines, cell cycle analysis revealed an accumulation of particles (sub-G1) with a small content of DNA only in T24 cells (Fig. 2), indicating that only in this cell line did the onset of apoptotic processes occur. In order to confirm this finding, we performed western blot analysis of PARP-1 and pro-caspase-3 and -9 . Proteolytic cleavage of specific proteins such as PARP-1 has been shown to occur in cells exposed to a number of apoptotic stimuli (23-25). EVOOE was found to induce apoptotic cell

Figure 2. EVOOE blocks cell cycle progression at G2/M stage. Distribution of T24 and 5637 cells in the different cell cycle compartments was analyzed by flow cytometry after $24 \mathrm{~h}$ in the absence or presence of various doses of EVOO phenolic extract following staining with PI. The panels show representative flow cytometry profiles obtained from three independent experiments. EVOOE, extra-virgin olive oil extract.

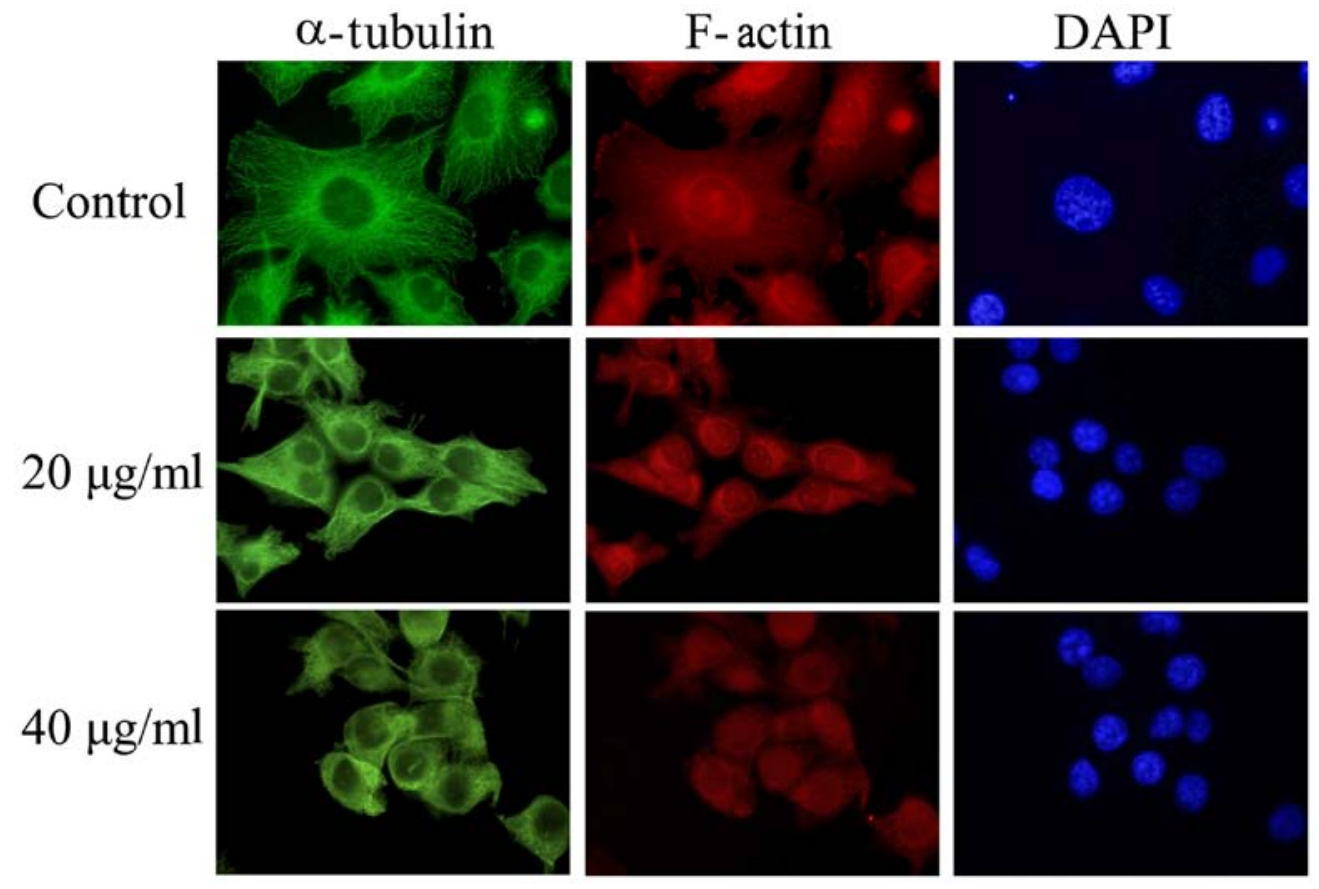

Figure 3. EVOOE exposure alters T24-cell morphology due to cytoskeleton remodelling. After $4 \mathrm{~h}$ of exposure to different EVOOE concentrations, the cells were subjected to immunofluorescence for F-actin (red color) and $\alpha$-tubulin (green color) staining. The images shown are representative immunofluorescence staining of three independent experiments. Magnification, x600. EVOOE, extra-virgin olive oil extract; F-actin, fibrous actin. 
$\mathrm{T} 24$

$\operatorname{EVOOE}(\mu \mathrm{g} / \mathrm{ml})$

PARP-1

Cleaved PARP-1

Pro-caspase-3

Pro-caspase-9

GAPDH $\begin{array}{llll}0 & 10 & 20 & 40\end{array}$
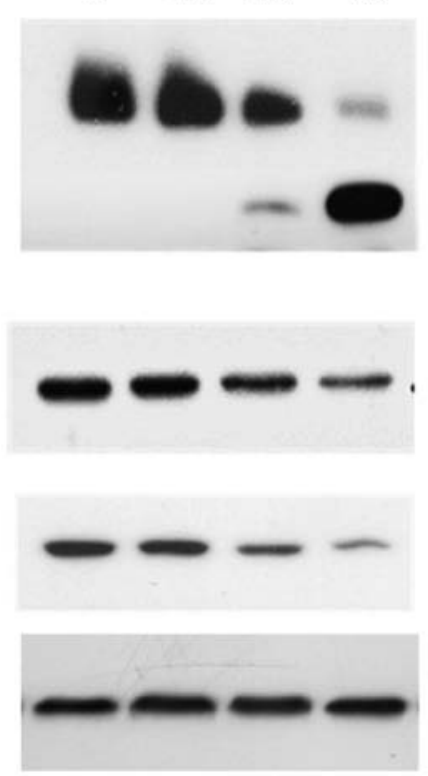

5637
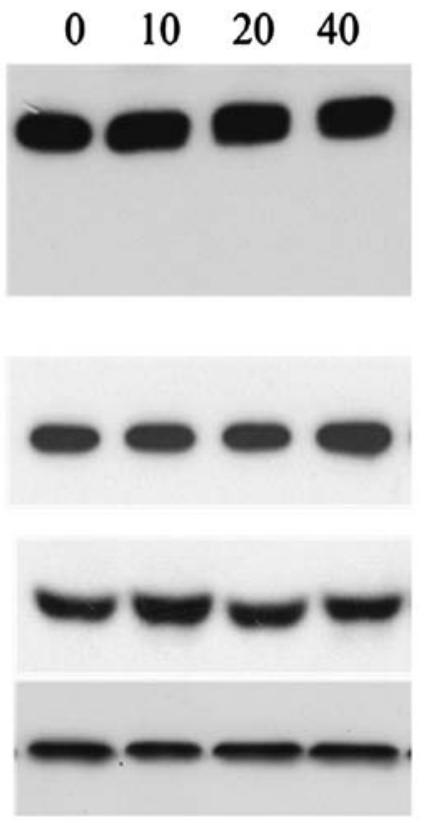

Figure 4. Western blot analysis of apoptosis-related proteins. After $24 \mathrm{~h}$ of exposure to different doses of EVOOE, the cell samples were subjected to western blot analysis to assess the cleavage of PARP-1 and of pro-caspase- 3 and -9 protein levels. EVOOE, extra-virgin olive oil extract.

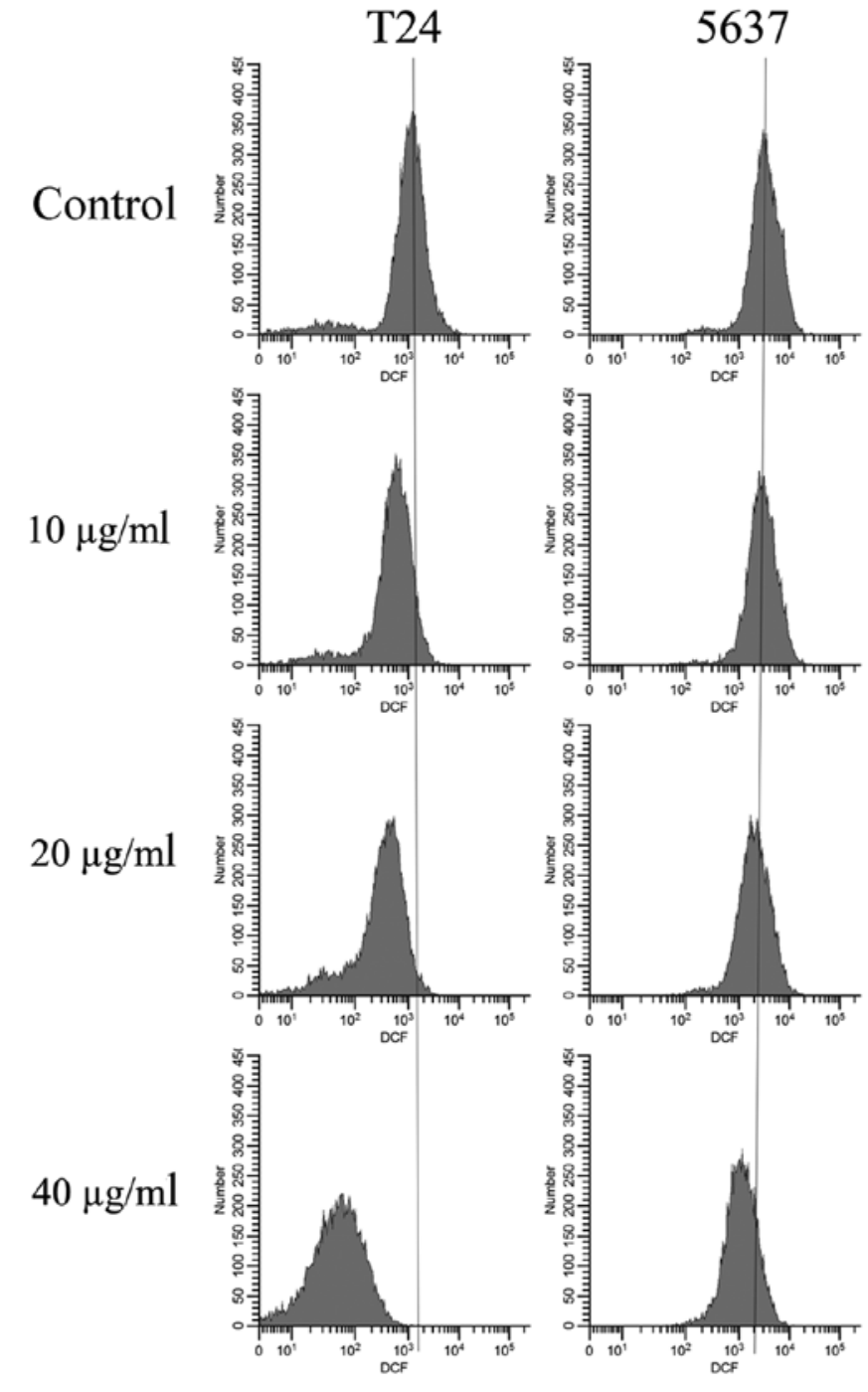

death only in T24 cells in a dose-dependent manner. Indeed, western blot analysis (Fig. 4) of the cleavage of PARP-1 showed a decrease in the full-size MW 116,000 fragment and an increase in the cleaved form within $24 \mathrm{~h}$ after the T24 cell treatment in a dose-dependent manner (Fig. 4). Western blot analysis of caspase- 3 and -9 also showed a marked decrease in their protein levels at higher doses of the polyphenol extract. Conversely to what was observed for T24 cells, the treatment of 5637 cells did not induce apoptosis, as is clearly visible from Fig. 4 that shows the lack of any PARP-1 cleavage.

EVOOE treatment decreases intracellular ROS production. The antioxidant effect of EVOOE was analyzed by determining intracellular ROS levels using cytofluorimetric analysis. After $1 \mathrm{~h}$ of treatment with different concentrations of EVOOE, the basal ROS production decreased in a dose-dependent manner as clearly evidenced by the leftward shift of fluorescence that became greater at increasing doses of the polyphenol extract (Fig. 5). The data analysis revealed a decrease in the mean T24-cell fluorescence ranging from $12 \%$ at $10 \mu \mathrm{g} / \mathrm{ml}$ to $64 \%$ at $40 \mu \mathrm{g} / \mathrm{ml}$. The same behavior was observed for the 5637 cells with a lesser antioxidant activity that became evident only at a dose of $40 \mu \mathrm{g} / \mathrm{ml}$ with a decrease in the mean cell fluorescence of $35 \%$.

Figure 5. EVOOE treatment reduces ROS production in both cell lines. After treatment with different concentrations of EVOOE, the cells were stained with DCF-DA and analyzed by flow cytometry. The histograms represent the mean cell fluorescence which is directly related to ROS content. The straight line indicates the mean fluorescence of the untreated cells. The panels show representative flow cytometry profiles obtained from three independent experiments. EVOOE, extra-virgin olive oil extract; DCF, 2',7'-dichlorofluorescein. 
A

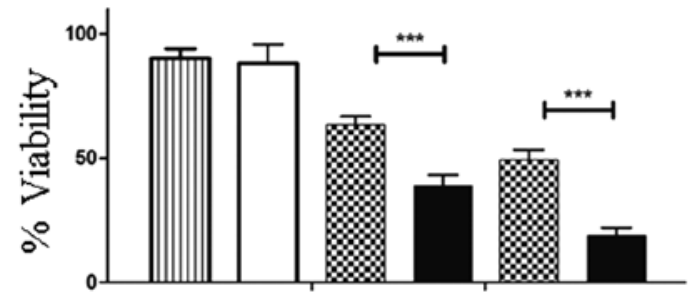

EVOOE

Mit. $20 \mu \mathrm{g} / \mathrm{ml}$

Mit. $100 \mu \mathrm{g} / \mathrm{ml}$

B

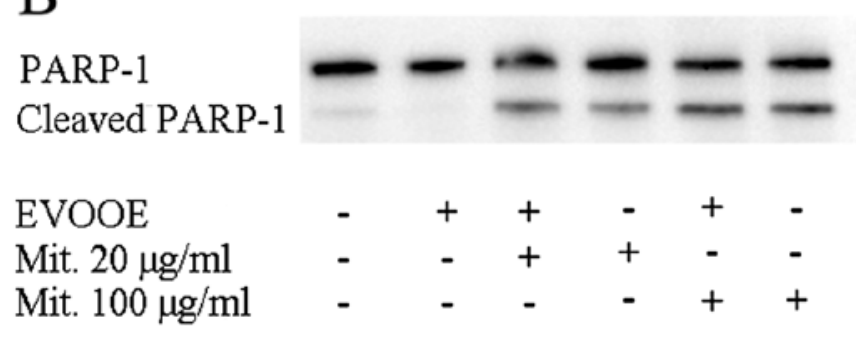

C
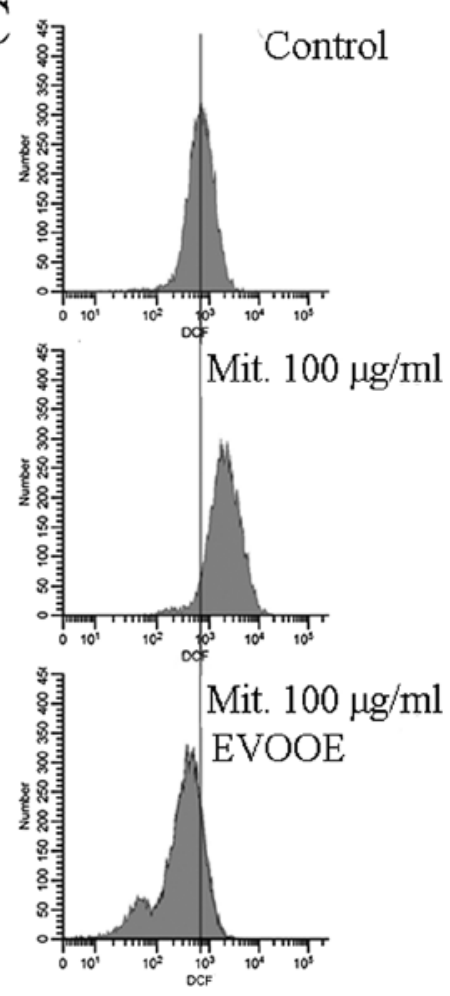

Figure 6. EVOOE co-treatment decreases mitomycin C (Mit.) cytotoxicity without affecting apoptotic cell death in BCa T24 cells. (A) T24 cell viability after $24 \mathrm{~h}$ of exposure to various doses of the chemotherapeutic in the presence or absence of $10 \mu \mathrm{g} / \mathrm{ml}$ EVOOE. Data are presented as means $\pm \mathrm{SD}$. ${ }^{* * *} \mathrm{P}<0.001$. (B) PARP-1 cleavage after $24 \mathrm{~h}$ of treatment of T24 cells as in (A). (C) Histograms representing the mean cell fluorescence which is directly linked to ROS content. The straight line indicates the mean fluorescence of the untreated cells. EVOOE, extra-virgin olive oil extract; BCa, bladder cancer.

EVOOE negatively affects mitomycin cytotoxicity. Mitomycin $\mathrm{C}$ is a methylazirinopyrroloindoledione antineoplastic antibiotic isolated from the bacterium Streptomyces caespitosus and other Streptomyces bacterial species. Mitomycin C generates oxygen radicals, alkylates DNA, and produces interstrand DNA cross-links, thereby inhibiting DNA synthesis. This drug is often used in the chemotherapy of $\mathrm{BCa}(26)$.

To evaluate the effect of the simultaneous exposure to the drug and EVOOE on cell viability, T24 cells were treated for $24 \mathrm{~h}$ with different doses of mitomycin in the presence or absence of the phenolic extract. In all the experiments, EVOOE was used at a dose of $10 \mu \mathrm{g} / \mathrm{ml}$ which was found to be non-toxic.

The data shown in Fig. 6A demonstrated that, at each concentration tested, the simultaneous treatment of EVOOE and mitomycin reduced the chemotherapeutic cytotoxicity. At a drug concentration of $100 \mu \mathrm{g} / \mathrm{ml}$ the cell viability increased from $20 \%$ when exposed to mitomycin alone to $50 \%$ with the co-treatment, and similar behavior was observed at all of the concentrations tested. The data showed that EVOOE reduced the mitomycin antiproliferative ability without affecting PARP-1 cleavage (Fig. 6B). To clarify the possible involvement of ROS inhibition in the decrease of the drug cytotoxicity we performed DCF assay and we found that mitomycin at the dose of $100 \mu \mathrm{g} / \mathrm{ml}$ strongly increased intracellular ROS production in T24 cells (Fig. 6C) whereas the co-treatment with EVOOE strongly reduced the ROS production with a considerable leftward shift of fluorescence.
EVOOE positively affects paclitaxel cytotoxicity. Paclitaxel is a mitotic inhibitor isolated from the bark of the Pacific yew tree, Taxus brevifolia and is named Taxol. This antineoplastic agent is indicated for the treatment of advanced carcinoma of the ovary, and other types of cancer including BCa (27). To perform our experiments, we treated T24 cells for $24 \mathrm{~h}$ with three different concentrations of paclitaxel alone or in association with $10 \mu \mathrm{g} / \mathrm{ml}$ of EVOOE. The obtained data are shown in Fig. 7A. Importantly, at each tested concentration, the addition of EVOOE strongly increased drug cytotoxicity. When we treated the cells with $5 \mathrm{nM}$ paclitaxel, the cell viability decreased from 75 to $25 \%$ in the presence of the EVOOE, and a similar response was observed at 20 and $100 \mathrm{nM}$. Notably, data analysis showed that exposure to $100 \mathrm{nM}$ paclitaxel exerted the same cytotoxicity as exposure to $5 \mathrm{nM}$ of the same drug when used in association with EVOOE. In order to evaluate the mechanism underlying the increased cell death, we performed western blot analyses to assess PARP-1 cleavage. As is clearly evident from Fig. 7B, the simultaneous treatment of paclitaxel and EVOOE strongly increased the protein cleavage at each tested concentration. Moreover the data from the DCF assay showed that paclitaxel alone did not induce oxidative stress, whereas EVOOE co-treatment reduced basal ROS production (Fig. 7C).

\section{Discussion}

The relevance of polyphenols in nutrition and their possible role as new drug candidates is well documented by the vast 

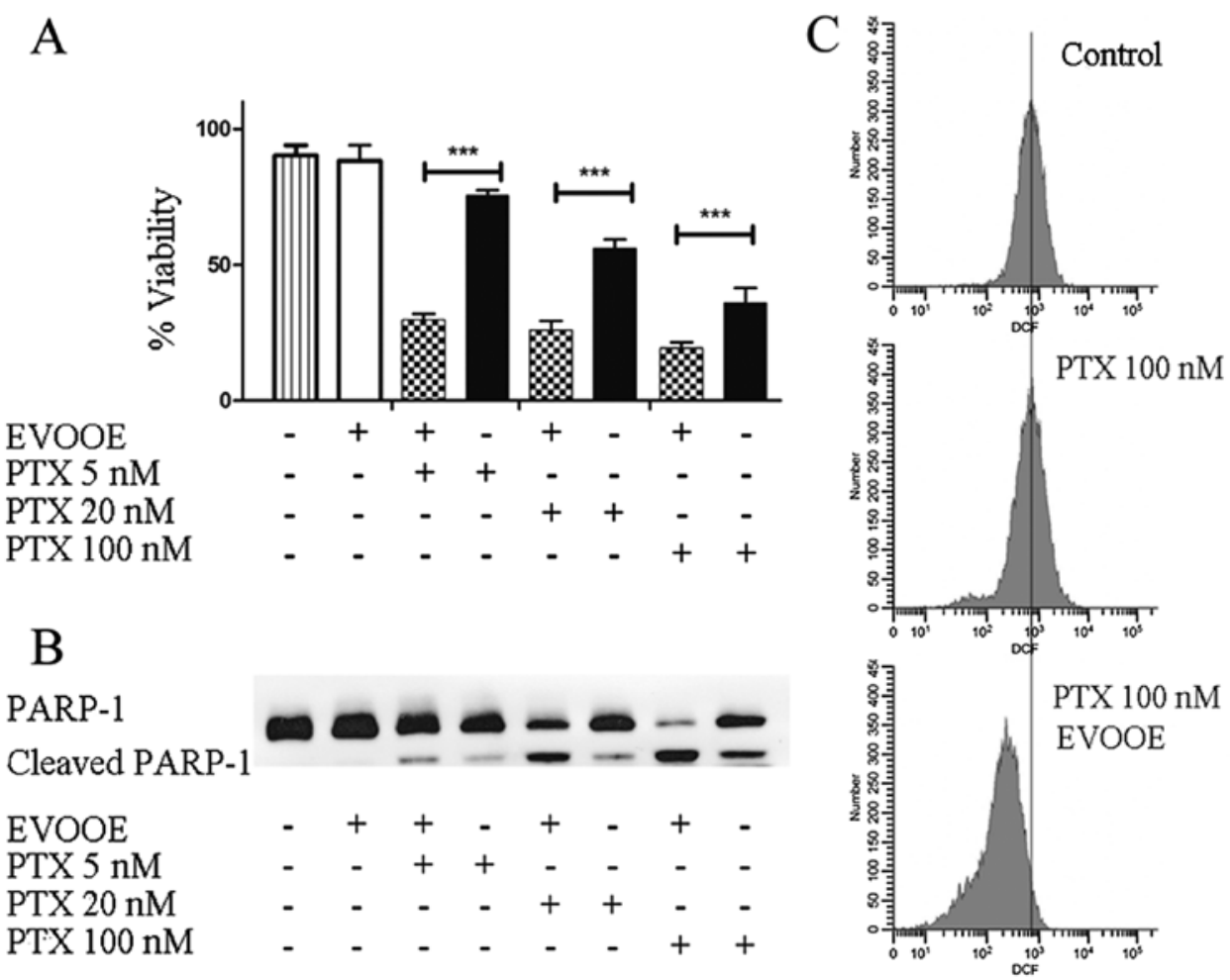

Figure 7. EVOOE co-treatment strongly increases paclitaxel (PTX)-induced apoptosis in BCa T24 cells. (A) T24 cell viability after $24 \mathrm{~h}$ of exposure to various doses of the chemotherapeutic in the presence or absence of $10 \mu \mathrm{g} / \mathrm{ml}$ EVOOE. Data are presented as means $\pm \mathrm{SD} .{ }^{* * *} \mathrm{P}<0.001$. (B) PARP-1 cleavage after $24 \mathrm{~h}$ of treatment of T24 cells as in (A). (C) Histograms represent the mean cell fluorescence which is directly linked to ROS content. The straight line indicates the mean fluorescence of the untreated cells. EVOOE, extra-virgin olive oil extract; $\mathrm{BCa}$, bladder cancer.

body of literature in recent years. Many studies have evaluated the effects of these molecules, either as single purified compounds or as crude extracts, on various pathophysiological processes, in particular as neuroprotective, cardioprotective, anti-inflammatory and anticancer agents.

The antioxidant activity of polyphenols was considered as the main mechanism of action of these compounds as anticancer agents, given the evidence that oxidative stress acts as a focal process in cancer development and progression (28). However, oxidative stress is only one of the many aspects of cancer pathophysiology which is a complex phenomenon and implies an alteration of many different signaling pathways not strictly related to intracellular redox equilibrium.

In recent years our group has focused on the effect of olive oil polyphenolic extracts on various cancer cell cultures, in particular on BCa cells. It is well known that after oral ingestion, polyphenols are excreted in urine and may reach concentrations high enough to exert a biological effect $(29,30)$. This phenomenon may explain the reduced incidence of $\mathrm{BCa}$ evidenced by epidemiological studies $(3,6)$.

Our previous study demonstrated that very low doses of total polyphenol extract from olive oil strongly affect bladder cell motility through a modulation of metalloprotease activity (20). In the present study, we evaluated whether the antiproliferative effect of EVOOE can modulate the toxicity of chemotherapeutic drugs commonly used in cancer treatment.

First, we evaluated the cell toxicity exerted by EVOOE on two different BCa cell lines, T24 and 5637, which are characterized by two different grades and are widely used as in vitro models (31-33) as they cover the more frequent subtypes of
BCa. 5637 cells well represent the E2F3/RB1 pathway due to amplification of 6p22.3, concomitant with loss of one copy of RB1 and mutation of the other copy. The T24 cell line belongs to the alternative pathway of FGFR3/CCND1 by mutated HRAS and over-represented CCND1 (34). Viability and clonogenic data indicated that $\mathrm{T} 24$ cells were more prone to the toxic effect of EVOOE compared to 5367 cells, and these data were further supported by cell cycle analyses which highlighted that both cell lines were arrested in the G2/M phase but with a different mechanism which resulted in apoptotic death only for T24 cells, with a consistent increase in the sub-G1 peak that was not evidenced in 5637 cells.

The data obtained from cell cycle analysis showed a similar mechanism of action of EVOOE, in fact both of the cell lines tested were subjected to growth arrest prior to mitosis. While performing viability experiments, we observed a considerable change in cell morphology, in both T24 and 5637 cells assuming a rounded shape even after a very short treatment. We hence performed immunofluorescence staining of the cytoskeletal proteins F-actin and $\alpha$-tubulin which are involved in the modulation of cell shape and mitosis. These experiments confirmed that after treatment with EVOOE there was a marked rearrangement of the cytoskeletal proteins, with a perinuclear accumulation of the actin filament. These data indicate that the arrest in the G2/M phase could be explained by the hampering of normal dynamics of the cytoskeleton and of the mitotic fuse. This evidence is in line with the findings of Hamdi and Castellon (35) who demonstrated that oleuropein treatment disrupted the organization of actin filaments thus altering the shape of cancer cells and their ability to proliferate. 
These data are also in line with the findings of Monti et al (36), who evidenced an interaction between oleocanthal and tau $(\tau)$ protein, a protein involved in the stabilization of microtubules, thereby modulating the plasticity of the cytoskeleton.

Apart from the arrest at the G2/M phase, the two cell lines show a different behavior regarding the accumulation of sub-G1 cell fragments, which are indicative of the onset of apoptotic processes. In order to evaluate the possible activation of apoptotic cell death, we investigated the cleavage of PARP-1 and the protein level of pro-caspase-3 and -9 following EVOOE treatment. Data confirmed the activation of apoptosis only in the T24 cells and only at high EVOOE doses, confirming the cytofluorimetric evidence. This different behavior of the two cell lines in response to EVOOE treatment, can be explained by the different mutational status in various key genes involved in cell proliferation regulation (34). The induction of programmed cell death only in T24 can also explain the different susceptibility to the cytotoxic action of EVOOE, as demonstrated by the difference in the decrease in viability observed in our experiments (Fig. 1).

A wide body of literature indicates that apoptosis may be responsible for polyphenol-mediated cell death. This effect has been attributed to ROS production induced by polyphenols in certain experimental conditions $(37,38)$. To clarify whether EVOOE may induce oxidative stress thus leading to apoptosis, we evaluated intracellular ROS production via a cytofluorimetric assay. Our data indicated that EVOOE was able to decrease the basal level of oxidative stress in the T24 cells and to a lower extent also in the 5637 cells. Hence, in our model the induction of apoptosis was not due to ROS production but had to be attributed to other molecular mechanisms such as the inhibition of pro-survival cell signaling pathways (37). In the last part of this study we evaluated the ability of low concentrations of EVOOE to improve the activity of different chemotherapeutics used in common clinical practice. It is of great interest to underline that current therapy in the management of $\mathrm{BCa}$ consists of bladder instillation of alchilant agents (39) that on the other hand exert unfavorable effects (40). In the present study we used an approach based on the comparison of viability since one of the two treatments given in co-association was always non-toxic (EVOOE up to $10 \mu \mathrm{g} / \mathrm{ml}$ ). As expected, we obtained different results based on the drug tested. Our data demonstrated that simultaneous treatment with mitomycin and EVOOE markedly decreased the cytotoxicity of the chemotherapeutic. These results are surprising only in part because it is well known that mitomycin exerts its toxic effect through the induction of oxidative stress and the antioxidant activity of the phenolic molecules may dampen its efficacy.

Conversely to what was observed for mitomycin, the addition of EVOOE to the paclitaxel treatment led to a strong increase in the drug toxicity with a marked induction of apoptotic cell death. These data are of great interest since the dose of EVOOE used for this experiment was non-toxic per se to the cells themselves, and this indicates a synergic effect in combination with paclitaxel. It can be hypothesized that the cytotoxicity enhancement of paclitaxel could be attributed to the alteration in gene expression occurring at low doses of phenolic extract, as observed in our previous study (20), that chemosensitize the cells by altering pathways involved in cell growth and proliferation such as platelet-activating factor (PAF) receptor signaling or PI3K signaling $(37,41,42)$.

There are controversial data concerning the role of antioxidant supplements during chemotherapy (43-45), however it is generally recommended to avoid the use of antioxidant food supplements and herbal products. Based on our experimental results, the polyphenolic extracts could be developed as potential adjuvants in combination with certain chemotherapeutic drugs which do not exert cytotoxicity through the induction of oxidative stress.

Collectively, our findings suggest that the potential adjuvant properties of EVOO phenols are related to the class of drugs used according to previously published data that point out how the adjunct of high doses of an antioxidant to chemotherapy does not always improve the pharmacological treatment (46). It is important to highlight that even if the mechanism of action of many chemotherapeutics and also radiotherapy is based in part on the production of free radicals $(44,45)$ there are also cytostatic drugs that do not exert their effects through oxidative stress mechanisms such as paclitaxel, vinca alkaloids, anthracycline and many others that can be improved by the use of antioxidant-rich supplements (43).

In conclusion, our data demonstrated that the combination of EVOOE and paclitaxel exhibited a markedly higher antiproliferative activity in vitro compared with each of these alone. To the best of our knowledge this is the first report that demonstrates how EVOO phenols markedly improve the activity of paclitaxel, and this evidence may pave the way for the study of new therapeutic strategies which exploit the synergy between drugs and nutraceuticals.

\section{Acknowledgements}

This study was supported by L.I.L.T. 'Lega Italiana per la Lotta contro i Tumori' section of Latina and 'Fondazione Terzo Pilastro - Italia e Mediterraneo'. The authors gratefully acknowledge 'CAPOL association' for providing the EVOO samples.

\section{References}

1. Goodison S, Rosser CJ and Urquidi V: Bladder cancer detection and monitoring: Assessment of urine- and blood-based marker tests. Mol Diagn Ther 17: 71-84, 2013.

2. Burger M, Catto JWF, Dalbagni G, Grossman HB, Herr H, Karakiewicz P, Kassouf W, Kiemeney LA, La Vecchia C, Shariat S, et al: Epidemiology and risk factors of urothelial bladder cancer. Eur Urol 63: 234-241, 2013.

3. Brinkman MT, Buntinx F, Kellen E, Van Dongen MC, Dagnelie PC, Muls E and Zeegers MP: Consumption of animal products, olive oil and dietary fat and results from the Belgian case-control study on bladder cancer risk. Eur J Cancer 47: 436-442, 2011.

4. Park SY, Ollberding NJ, Woolcott CG, Wilkens LR, Henderson BE and Kolonel LN: Fruit and vegetable intakes are associated with lower risk of bladder cancer among women in the Multiethnic Cohort Study. J Nutr 143: 1283-1292, 2013.

5. Ros MM, Bueno-de-Mesquita HB, Kampman E, Aben KK, Büchner FL, Jansen EH, van Gils CH, Egevad L, Overvad K, Tjønneland A, et al: Plasma carotenoids and vitamin C concentrations and risk of urothelial cell carcinoma in the European Prospective Investigation into Cancer and Nutrition. Am J Clin Nutr 96: 902-910, 2012.

6. Zamora-Ros R, Sacerdote C, Ricceri F, Weiderpass E, Roswall N, Buckland G, St-Jules DE, Overvad K, Kyrø C, Fagherazzi G, et al: Flavonoid and lignan intake in relation to bladder cancer risk in the European Prospective Investigation into Cancer and Nutrition (EPIC) study. Br J Cancer 111: 1870-1880, 2014. 
7. Psaltopoulou T, Kosti RI, Haidopoulos D, Dimopoulos M and Panagiotakos DB: Olive oil intake is inversely related to cancer prevalence: A systematic review and a meta-analysis of 13,800 patients and 23,340 controls in 19 observational studies. Lipids Health Dis 10: 127, 2011.

8. Giacosa A, Barale R, Bavaresco L, Gatenby P, Gerbi V, Janssens J, Johnston B, Kas K, La Vecchia C, Mainguet P, et al: Cancer prevention in Europe: The Mediterranean diet as a protective choice. Eur J Cancer Prev 22: 90-95, 2013.

9. Bulotta S, Celano M, Lepore SM, Montalcini T, Pujia A and Russo D: Beneficial effects of the olive oil phenolic components oleuropein and hydroxytyrosol: Focus on protection against cardiovascular and metabolic diseases. J Transl Med 12: 219 , 2014.

10. Cárdeno A, Sánchez-Hidalgo M and Alarcón-de-la-Lastra C: An up-date of olive oil phenols in inflammation and cancer: Molecular mechanisms and clinical implications. Curr Med Chem 20: 4758-4776, 2013.

11. Nomikos T, Fragopoulou E and Antonopoulou S: Food ingredients and lipid mediators. Curr Nutr Food Sci 3: 255-276, 2007

12. Nasopoulou C, Gogaki V, Stamatakis G, Papaharisis L, Demopoulos CA and Zabetakis I: Evaluation of the in vitro anti-atherogenic properties of lipid fractions of olive pomace, olive pomace enriched fish feed and gilthead sea bream (Sparus aurata) fed with olive pomace enriched fish feed. Mar Drugs 11: 3676-3688, 2013.

13. Masci A, Coccia A, Lendaro E, Mosca L, Paolicelli P and Cesa S: Evaluation of different extraction methods from pomegranate whole fruit or peels and the antioxidant and antiproliferative activity of the polyphenolic fraction. Food Chem 202: 59-69, 2016.

14. Hernáez Á, Remaley AT, Farràs M, Fernández-Castillejo S, Subirana I, Schröder H,Fernández-Mampel M, Muñoz-Aguayo D, Sampson M, Solà R, et al: Olive oil polyphenols decrease LDL concentrations and LDL atherogenicity in men in a randomized controlled trial. J Nutr 145: 1692-1697, 2015.

15. Martín-Peláez S, Covas MI, Fitó M, Kušar A and Pravst I: Health effects of olive oil polyphenols: Recent advances and possibilities for the use of health claims. Mol Nutr Food Res 57: 760-771, 2013.

16. Jayasena T, Poljak A, Smythe G, Braidy N, Münch G and Sachdev P: The role of polyphenols in the modulation of sirtuins and other pathways involved in Alzheimer's disease. Ageing Res Rev 12: 867-883, 2013.

17. Vauzour D, Rodriguez-Mateos A, Corona G, Oruna-Concha MJ and Spencer JPE: Polyphenols and human health: Prevention of disease and mechanisms of action. Nutrients 2: 1106-1131, 2010.

18. Zhao B, Ma Y, Xu Z, Wang J, Wang F, Wang D, Pan S, Wu Y, Pan H, Xu D, et al: Hydroxytyrosol, a natural molecule from olive oil, suppresses the growth of human hepatocellular carcinoma cells via inactivating AKT and nuclear factor-kappa B pathways. Cancer Lett 347: 79-87, 2014.

19. Casaburi I, Puoci F, Chimento A, Sirianni R, Ruggiero C, Avena P and Pezzi V: Potential of olive oil phenols as chemopreventive and therapeutic agents against cancer: A review of in vitro studies. Mol Nutr Food Res 57: 71-83, 2013.

20. Coccia A, Bastianelli D, Mosca L, Monticolo R, Panuccio I, Carbone A, Calogero A and Lendaro E: Extra virgin olive oil phenols suppress migration and invasion of T24 human bladder cancer cells through modulation of matrix metalloproteinase-2. Nutr Cancer 66: 946-954, 2014.

21. Impellizzeri $\mathbf{J}$ and Lin $\mathrm{J}$ : A simple high-performance liquid chromatography method for the determination of throat-burning oleocanthal with probated antiinflammatory activity in extra virgin olive oils. J Agric Food Chem 54: 3204-3208, 2006.

22. Bulotta S, Corradino R, Celano M, D'Agostino M, Maiuolo J, Oliverio M, Procopio A, Iannone M, Rotiroti D and Russo D Antiproliferative and antioxidant effects on breast cancer cells of oleuropein and its semisynthetic peracetylated derivatives. Food Chem 127: 1609-1614, 2011.

23. Chen H, Landen CN, Li Y, Alvarez RD and Tollefsbol TO: Epigallocatechin gallate and sulforaphane combination treatment induce apoptosis in paclitaxel-resistant ovarian cancer cells through hTERT and Bcl-2 down-regulation. Exp Cell Res 319: 697-706, 2013.

24. Yang K, Zheng XY, Qin J, Wang YB, Bai Y, Mao QQ, Wan Q, Wu ZM and Xie LP: Up-regulation of p21WAF1/Cip1 by saRNA induces G1-phase arrest and apoptosis in T24 human bladder cancer cells. Cancer Lett 265: 206-214, 2008.
25. Yuan SSF, Chang HL, Chen HW, Kuo FC, Liaw CC, Su JH and Wu YC: Selective cytotoxicity of squamocin on T24 bladder cancer cells at the S-phase via a Bax-, Bad-, and caspase-3-related pathways. Life Sci 78: 869-874, 2006.

26. Nargund VH, Tanabalan CK and Kabir MN: Management of non-muscle-invasive (superficial) bladder cancer. Semin Oncol 39: 559-572, 2012.

27. Brighenti M, Passalacqua R, Arnaudi R, Potenzoni M, Donini M, Liguigli W, Poli R, Lazzarelli S, Panni S and Curti A: High rate of complete remission (CR) using two sequential, dose-dense regimens of cisplatin, gemcitabine, and paclitaxel (CGP) followed by HD-MVAC in patients with metastatic bladder cancer (mBC). Eur J Cancer 47: S516, 2011.

28. Mileo AM and Miccadei S: Polyphenols as modulator of oxidative stress in cancer disease: New therapeutic strategies. Oxid Med Cell Longev 2016: 6475624, 2016.

29. Vissers MN, Zock PL, Roodenburg AJC, Leenen R and Katan MB: Olive oil phenols are absorbed in humans. J Nutr 132: 409-417, 2002.

30. Miró-Casas E, Covas MI, Fitó M, Farré-Albadalejo M, Marrugat $\mathbf{J}$ and de la Torre R: Tyrosol and hydroxytyrosol are absorbed from moderate and sustained doses of virgin olive oil in humans. Eur J Clin Nutr 57: 186-190, 2003.

31. Zhou XU, Qi L, Tong S, Cui YU, Chen J, Huang T, Chen Z and $\mathrm{Zu} \mathrm{XB}$ : miR-128 downregulation promotes growth and metastasis of bladder cancer cells and involves VEGF-C upregulation. Oncol Lett 10: 3183-3190, 2015.

32. Liao YX, Zeng JM, Zhou JJ, Yang GH, Ding K and Zhang XJ: Silencing of RTKN2 by siRNA suppresses proliferation, and induces $\mathrm{G} 1$ arrest and apoptosis in human bladder cancer cells. Mol Med Rep 13: 4872-4878, 2016.

33. Pacini L, De Falco E, Di Bari M, Coccia A, Siciliano C, Ponti D, Pastore AL, Petrozza V, Carbone A, Tata AM, et al: M2 muscarinic receptors inhibit cell proliferation and migration in urothelial bladder cancer cells. Cancer Biol Ther 15: 1489-1498, 2014.

34. Pinto-Leite R, Carreira I, Melo J, Ferreira SI, Ribeiro I, Ferreira J, Filipe M, Bernardo C, Arantes-Rodrigues R, Oliveira P, et al: Genomic characterization of three urinary bladder cancer cell lines: Understanding genomic types of urinary bladder cancer. Tumour Biol 35: 4599-4617, 2014.

35. Hamdi HK and Castellon R: Oleuropein, a non-toxic olive iridoid, is an anti-tumor agent and cytoskeleton disruptor. Biochem Biophys Res Commun 334: 769-778, 2005.

36. Monti MC, Margarucci L, Tosco A, Riccio R and Casapullo A: New insights on the interaction mechanism between tau protein and oleocanthal, an extra-virgin olive-oil bioactive component. Food Funct 2: 423-428, 2011.

37. Yan CM, Chai EQ, Cai HY, Miao GY and Ma W: Oleuropein induces apoptosis via activation of caspases and suppression of phosphatidylinositol 3-kinase/protein kinase B pathway in HepG2 human hepatoma cell line. Mol Med Rep 11: 4617-4624, 2015.

38. Sun L, Luo C and Liu J: Hydroxytyrosol induces apoptosis in human colon cancer cells through ROS generation. Food Funct 5 : 1909-1914, 2014

39. Raghavan D, Burgess E, Gaston KE, Haake MR and Riggs SB: Neoadjuvant and adjuvant chemotherapy approaches for invasive bladder cancer. Semin Oncol 39: 588-597, 2012.

40. Branchereau J, Luyckx F, Hitier M, Karam G, Bouchot O and Rigaud J: Bladder necrosis after an immediate post-operative mitomycin C instillation. Prog Urol 21: 151-153, 2011 (In French).

41. Onuchic AC, Machado CM, Saito RF, Rios FJ, Jancar S and Chammas R: Expression of PAFR as part of a prosurvival response to chemotherapy: A novel target for combination therapy in melanoma. Mediators Inflamm 2012: 175408, 2012.

42. Melnikova V and Bar-Eli M: Inflammation and melanoma growth and metastasis: The role of platelet-activating factor (PAF) and its receptor. Cancer Metastasis Rev 26: 359-371, 2007.

43. Nechuta S, Lu W, Chen Z, Zheng Y, Gu K, Cai H, Zheng W and Shu XO: Vitamin supplement use during breast cancer treatment and survival: A prospective cohort study. Cancer Epidemiol Biomarkers Prev 20: 262-271, 2011.

44. Moss RW: Do antioxidants interfere with radiation therapy for cancer? Integr Cancer Ther 6: 281-292, 2007.

45. D'Andrea GM: Use of antioxidants during chemotherapy and radiotherapy should be avoided. CA Cancer J Clin 55: 319-321, 2005.

46. Gröber U: Antioxidants and other micronutrients in complementary oncology. Breast Care (Basel) 4: 13-20, 2009. 